\title{
Call for Information about Microcomputers
}

Educational Measurement: Issues and Practice wishes to publish articles, features and other useful information about the application(s) of microcomputers to testing, measurement, and assessment. We are developing a special issue devoted to this topic. We also plan to publish relevant articles and features regularly in various issues of $E M$.

In order to develop these plans, I need your help. I need information about software that has been developed and/or is being developed for using microcomputers to enhance testing; I need information about persons who are developing such software for microcomputers; I need information about continuing "sources" of information; I need suggestions of persons who could contribute to this effort. Please keep in mind that $E M$ is a publication focusing on practices and applications, rather than on theory or on hardware, and that EM is a publication focusing on testing and measurement, rather than on curriculum and instruction. With these caveats in mind, please fill out the form on this page and send it to me no later than July 1, 1983.

\section{Microcomputers and Testing}

Frank Womer

Software

Name of Product

Function of Product

Computer Requirements

Address for Information about Product

Individuals Professionally Active in the Area of Microcomputers in Testing

Name

Address

Area of Specialization (describe)

Projects Addressing Microcomputer Applications in Testing, Measurement, Assessment

Name of Project

Organization/Person Conducting Project

Address

Purpose of Project

Project Beginning and Ending Dates

Additional information/comments/suggestions will be welcome.

Feel free to reproduce this form for others. NCME membership is NOT a requisite.

Frank B. Womer, Editor,

Educational Measurement: Issues and Practice, The University of Michigan, School of Education, Bureau of School Services,

610 East University, Ann Arbor, Michigan 48109 